\title{
Problem of Assessing the Investment Attractiveness of Risk Projects for Developing Artificial Intelligence
}

\author{
Irina Reshetnikova, Olga Yanina, Larisa Semenova, Lesya Bozhko, Oleg Veselitsky
}

\begin{abstract}
The article discusses the problem of assessing the investment attractiveness of risk projects for developing artificial intelligence, the methods of such assessment and their features. It is shown that due to the lack of relevant statistical, financial, operational information, the models and methods of investment valuation are, for the most part, subjective. The use of only one model or method of assessing investment attractiveness in the field of the development of artificial intelligence projects is insufficient, while the complex use without taking into account systemic aspects is likewise not sufficiently substantiated.

To solve the existing problem, it is proposed to comprehensively use the available capabilities of the method of functional cost analysis (FSA), the essence of which is that the development project is decomposed into separate functions, and the necessary resources are measured and fixed for each function. Analysis of the functions of the object and the costs of the implementation of the functions makes it possible to identify the most acceptable variant of the object from the position of its functional content.

At the same time, the article considers the possibility of using the functional-cost analysis method in the evaluation, the essence of which is that the development project is decomposed into separate functions, and for each function, all necessary resources are measured and fixed. An analysis of the object's functions and their costs will help to identify the most economical version of a risky investment project from its functional content.

It is reasonably noted that the main resources to support and promote the development of innovative projects are venture companies that invest considerable funds both at the initial stages and at the stages of development and expansion of projects. The amount of financial resources coming from business angels, crowdfunding and business accelerators is much smaller and goes mainly to the initial stages of project implementation.
\end{abstract}

Keywords : artificial intelligence development project, investment attractiveness, valuation methods, functional-cost analysis, venture financing, business-angels, crowdfunding.

\section{INTRODUCTION}

The key to the successful development of the state is the availability of innovations that can simplify and facilitate the life of both an individual and society as a whole. Today, startups, IT projects, artificial intelligence development

Revised Manuscript Received on September 25, 2019

Irina Reshetnikova, Tyumen Industrial University, Tyumen, Russian Federation

Olga Yanina, Russian state social University, Balashikha, Russian Federation

Larisa Semenova, Bashkir State Medical University of the Ministry of Health of the Russian Federation, Ufa, Russian Federation

Lesya Bozhko, Emperor Alexander I St. Petersburg State Transport University, Saint Petersburg, Russian Federation

Oleg Veselitsky, Moscow City Government University of Management Moscow, Moscow, Russian Federation

projects, etc. are considered innovative projects. Leading global companies and companies wishing to have high competitiveness pay attention to intelligent solutions for production. The introduction of new systems, software and network setup, the purchase of equipment require not only attracting a significant amount of resources but also have many advantages (automation, data analysis, minimizing operational errors, etc.).

Like any business, such projects need investments, especially at the initial stages of implementation, so the problem of finding sources of financing is highly relevant for them. Issues related to the search, attraction, and distribution of financial resources to support and develop risky projects of artificial intelligence are of particular relevance, given the rapid growth in their number, scale and market demand.

To get help in raising funds, entrepreneurs formally have the opportunity to turn to business incubators, accelerators, venture funds, and private investors. However, practice shows that these sources are insufficient, and the activity of investment and innovation activity remains low (Loktionova, 2017). This is partly due to the lack of a clear methodology for determining the investment attractiveness of projects related to artificial intelligence and the perception of these projects as a potential bubble (similar to the Dot-Com Bubble) (de Mello \& de Souza, 2019; Loktionova \& Yanina, 2019).

At the planning stage of a new project, investors are interested in the amount of resources needed for implementation and the potential profitability of the project. To do this, a preliminary assessment of the project is carried out, where on its basis a decision is made on investing in the project or on its rejection. It is also important to identify risks, for the assessment of which different methods are used. All of them have strengths and weaknesses, but to choose a specific method for assessing the investment attractiveness of a project, an understanding of all existing methods is necessary.

\section{LITERATURE REVIEW}

According to the commercial database of innovative companies Crunchbase (containing information on more than 500.000 legal entities located in 199 countries), investors in artificial intelligence (AI) development projects are usually private joint-stock companies, venture companies and angel investors (OECD, 2018). The total amount of investments and the global number of transactions have increased significantly since 2011, but investment models continue to vary by region: for China, it is typical to support a small number of large-scale projects requiring significant

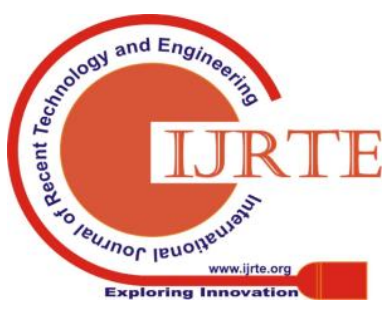


investment; the EU is characterized by the support of a large number of small projects; the United States is characterized by a steady increase in the number of large investments. In total, artificial intelligence development projects in the database represented 689 of 2436 existing innovative projects. At the same time, AI development projects, for the most part, focus on the development of computer vision, analytics for forecasts, natural language processing, autonomous vehicles, intelligent systems, and virtual assistants. Most of the projects relate to the development of autonomous vehicles. To develop risky projects of artificial intelligence, neural networks, deep learning, reinforcement learning are used. It should be noted that $26 \%$ of investment transactions for the launch of $\mathrm{AI}$ in the specified database do not report the amount of investment received for the implementation of the project.

Investments in risky projects for developing artificial intelligence are traditionally considered in the context of assessing the investment attractiveness of startups. To evaluate them, the following models and methods were developed:

- The method of profit margin, which is quite popular, but has significant shortcomings in the assessment. For example, the P/E coefficient (function of cash flows) - this method does not take into account the subjective nature of the ROR rate (since the target rate of return is set by the investor himself, this means a direct opportunity to change the final cost of the project) (Köhn, 2017);

- The method of discounting cash flows allows evaluating based on cash flow indicators for the period of investor participation in it. Since such projects, as a rule, do not have a financial history, cash flows here are exclusively interval prognostic in nature (Köhn, 2017);

- The AVE MARIA method (Acquisition, Value, Engagement, Monetization, Retention, Intellectual Property) is descriptive and answers the questions: what is the audience of the project, what is its size, how can it be described and characterized; what is the cost of attracting a user (client) in different channels; what is the list of preferred actions for the active and passive user, as well as secondary, additional actions; how the number and activity of users will be converted into project revenue; how to make the client return and turn him into a regular user; how the company protects itself from the machinations of competitors and the fact that the employee leaves and opens a competing company. The AVE MARIA method does not provide estimated characteristics of the projects, but is convenient for comparing them when choosing the preferred direction of investment;

- $\quad$ The method of valuing the potential audience can be used to assess the future value of the project, the success of which is closely related to a specific audience of customers (Köhn, 2017);

Berkus D. (2012) evaluates the project according to the following parameters: promising idea, implemented prototype, strategically important connections, product promotion on the market. The method focuses on the method of evaluating projects at an early stage. The primary value of the Berkus D. method is that it gives confidence to the investor in the prospects of the project, the assessment of which is subjective. The model was improved by Payne B. (2011; Berkus, 2016), who put the priority on the quality of the management team and indicators of the project implementation environment: the size of the potential market, the level, and structure of market competition in the selected segment, the degree of protection of intellectual property;

- $\quad$ The scoring method is based on the assessment and comparison of the project with the average performance of similar projects. At the same time, the importance of the management team and the size of the market exceed the importance of products and technologies. The disadvantage of this method is its subjective nature ranking of factors of influence during the assessment (Payne, 2017);

- The venture capital method is based on the assessment of Return on Investment as the ratio of Terminal Value to Post-money Valuation (evaluation after receipt of funds) (Payne, 2011b);

- $\quad$ The Risk Factor Summation Method considers various types of risks: Management risk, Stage of the business, Legislation/Political risk, Manufacturing risk, Sales and marketing risk, Funding/Capital raising (managerial risk; risk associated with a certain stage of the business; risk of change legislation; risk of unsuccessful marketing and low sales; risk of insufficient funding) (Payne, 2011c).

It should be noted that due to the lack of relevant statistical, financial, operational information, as well as information on previously implemented developments, investment assessment models are, for the most part, subjective. Researchers (Kunitsyna \& Khalyavskaya, 2016; Kirshina \& Lebedinsky, 2019) showed that assessment should be carried out using a combination of several methods, since individual assessment methods are, for the most part, empirical, without an intelligible theoretical and statistical basis.

In (Carson, 2018), the results of a survey of business angels, project developers, and venture businessmen are presented, where it is established that for these categories of participants in investment processes, risk factors and their importance differ significantly when assessing the investment attractiveness of a project. In (Zhong et al., 2018), it was shown that investment verification and project evaluation largely depend on the personal experience of investors. The authors of the study (Zhong et al., 2018) propose a model for determining the amount of financing for a particular project which provides for the presence of a probabilistic hidden factor for the overall assessment of investment preferences of all investors and is based on retrospective statistical information.

Individual investors ("business angels") are prepared for big risks, they make emotional decisions based on whether they like the project or not (Ederman et al., 2017). Typically, business angels do not advertise their activities, so it is impossible to assess the real size of the informal investment market. Venture funds, on the other hand, take responsibility for their investors, therefore they take a more rational approach to making decisions: they evaluate how the market reacts to new products and conduct a full audit of a specific project. In (Cantamessa et al., 2018), the authors analyzed 214 reports on completed projects and concluded that the key factor in the failure of the project is the lack of a business development strategy. It was shown in (Damodaran, 2009) that even those young development companies that are profitable depend on private capital, the initial savings of owners, venture capital and the 
capital of private investors. Besides, in the first years after the start of operations, such companies generate negative cash flow. In this regard, many standard methods that are used to estimate cash flows, growth rates, and discount rates do not apply to such companies. The quantitative Equbot Model developed by Equbot LLC and Watson to assess the investment value of artificial intelligence development projects is presented in (Saint-Pierre, 2017). This model can be applied in an applied way to evaluate AI development projects in the financial sector. In (Ayukawa, 2012), an extended Hart and Holmstrom model is presented, which, when assessing, considers individual investment transactions, rather than company assets. It is assumed that the investment attractive are those transactions that include not only profit but also private benefits. Private benefits include the benefits of learning through experimentation and collaboration, but this model has not received sufficient experimental verification. In (Alamsyah \& Nugroho, 2018), it was shown that crowdfunding financing could be an alternative to finding an individual investor. This method of financing is becoming very popular today. The difficulty lies in the fact that the project is obliged to interest a significant number of small investors, even though it cannot have predicted indicators of investment attractiveness. Thus, to obtain company financing, it is necessary to actively implement a marketing strategy to promote the project.

Thus, summarizing the research in the field of assessing investment attractiveness, it should be noted that the problem of investing in risky projects of developing artificial intelligence (AI) has some features:

- lack of criteria for applying methods to assess the investment attractiveness of projects at various intermediate stages of development;

- the need for significant material and labor costs for the development and implementation of projects;

- uncertainty of the target audience of projects;

- limited financial, operational information at the stage of development of the technical specifications of projects;

- the high final cost of projects and their scale, which narrows the potential circle of buyers, since such projects are often not relevant in small and medium-sized businesses;

- the need to adapt projects to broaden market niches.

The purpose of this study is to assess the investment attractiveness of artificial intelligence development projects, as well as to identify the features of the implementation of investing using various resource recipients.

\section{RESEARCH RESULTS}

The difference between an AI development project and a startup is that a startup is a holistic project that will result in some product, computer application, etc., and the AI project is highly specialized and developed for further work with any application, to automate the production process, data processing, etc. That is, the AI development project is created for the finished product or process, therefore, before starting the development of such a project, it is advisable to have an agreement on sales, implementation and future use. Otherwise, the development of an AI project is highly risky. For example, the review (Mannar, 2019) proposes a phased approach to identifying risks and profitability of AI development projects. This approach makes it possible to assess the size and risk of future implementations. The approach includes three stages:

- analysis of the scope of the AI development project. It is necessary to analyze AI projects that are already used in this area, and also to develop a trial small project.

- application of several assessment methods.

- simulation modeling.

Using this approach, the following projects have been implemented:

- the company owning the forest, using AI projects, determined the planting sites for new seedlings to increase the overall productivity of the forest area;

- AI project designed for law enforcement agencies whose goal was to prevent recidivism. For the test group, the AI model determined the risk factors for potential reoffending, and the authorities took a targeted response;

- the industrial design of a predictive maintenance model for critical equipment. The model showed that machines can be serviced less frequently safely, resulting in significant business savings. However, if these forecasts turn out to be incorrect, this can lead to a long-term outage or damage to key assets, so the use of simulation is necessary.

In general, the model of assessing investment attractiveness boils down to the following (Figure 1):

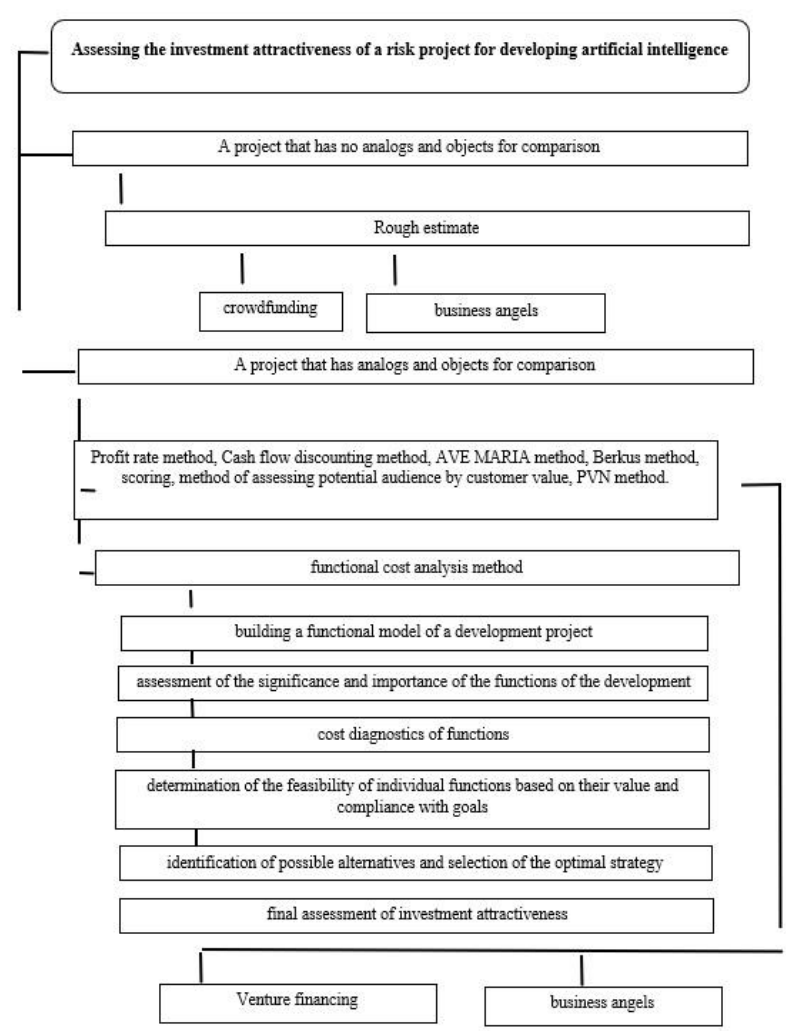

Figure 1. Diagram for assessing the investment attractiveness of a risk project for developing artificial intelligence and preferred financing methods

In the case of a risky project that has no analogues, there are no objects of comparison for it, so the process of assessing investment attractiveness is difficult. In general, a preliminary assessment of a project can be carried out using the methods described above, or using various Internet calculators (Equidam, AngelList, IPOboard, Starting

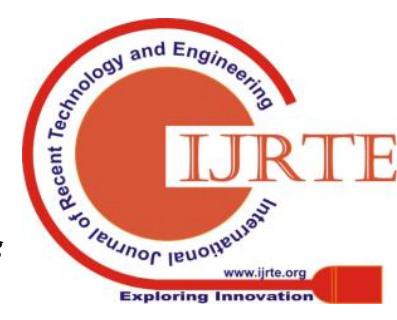


Costs Calculator, Calculate Total Startup Fund, Startup Valuation Calculator).

In this regard, the assessment is carried out approximately and financing can be carried out either through crowdfunding or through business angels.

Crowdfunding is the collective work of people (donors) who voluntarily pool funds or other resources, in most cases via the Internet, to support the efforts of other people or organizations (recipients). To implement these projects, the goal must first be stated, the necessary amount of money must be determined, all costs calculated, and during the fundraising process, the information should be open to all comers. In addition, crowdfunding platforms help to answer the question: Do users need a future product, which means that it has market potential and a commercially successful future?

Business angel is a private person with capital who invests small (compared to venture capital funds) cash for a share of ownership in projects. As a rule, a business angel is a person who himself was or still is an entrepreneur. He is led not only by the opportunity to increase his investment but also by his interest. Such a person, as a rule, not only finances the project and waits until it starts to make a profit, but also provides technical and consulting assistance (especially if the project chosen by the business angel works in the direction in which it achieved success). The business angels are partly reminiscent of venture capital investments. Thus, in that, and another case, investors are dealing with high-risk investments. They do not receive any collateral or guarantees from business angels, therefore they can only rely on experience, intuition and a competent approach to financial transactions. The success of such projects is facilitated by the joint teamwork of investors and project creators. The long-term goal of business angels is to develop investment projects and increase their value. The business angels receive their income mainly from the sale of their share in the company during its market success and capitalization growth. The main disadvantage of cooperation with business angels is their direct intervention in project management, which may not coincide with the vision of top managers and project developers, which creates the threat of termination of funding at any stage of project development.

The source of additional financial support for projects is often business accelerators (business incubators), which are both institutions and their intensive development programs through mentoring, training, financial and expert support in exchange for a share in the company's capital. A characteristic feature of accelerators is that they offer a structured program that will help the team work out its business model, acquire the necessary business connections and increase sales. The main difference between accelerators and business incubators is the acceleration program, which lasts from 3 months to six months (in a business incubator, the program can last from 1 to 4 years). Often, business accelerators work in conjunction with a venture fund (the fund gives investments, the accelerator - an educational program, networking, infrastructure)

In the case of a risk project that has analogs, it is necessary to use the various assessment methods discussed above. In this case, this expensive project competes with similar projects, which simplifies the methods of its evaluation and at the same time increases the risk. At the same time, it is advisable to develop a project in the presence of a buyer, an agreement, etc., that is, an AI project as a component of an integrated project. Otherwise, financing can be made by attracting business angels, business accelerators, venture capital funds, and rarely crowdfunding.

Venture capital investments made in the early stages of project development are, in some cases, practically the only source of financing, especially in projects with an innovative focus, which are characterized by high market risks. The degree of development of venture investment determines the development of innovative entrepreneurship, which means that it characterizes the growth rate of the economy and the state of its competitiveness, and also indicates the opportunities for sustainable development of entrepreneurial structures.

A key aspect of the use of investment attractiveness assessment methods is the assessment of the projected effect of the project. Even though the considered methods use predicted values, the reliability of numerical estimates may be called into question by investment decision-makers.

It should be separately considered the possibility of applying the method of functional cost analysis (FSA) to assess the effectiveness of project development, where the development project is decomposed into separate functions, and for each function, the necessary resources are measured and fixed. Analysis of the functions of the object and the costs of the implementation of the functions makes it possible to identify the most acceptable variant of the object from the position of its functional content (Mezentsev \& Preobrazhenskaya, 2003).

The goal of the FSA development process is to find reserves to reduce costs for the implementation of functions in a specified period with a certain quality of the project, which allows evaluating the effect of the implementation of the project in terms of minimizing the costs of its development and implementation.

The principles underlying the FSA (functional approach, development teamwork, multivariance, the use of cost criteria in evaluating technical solutions) are reflected in the methodology for its implementation. It includes some successively carried out steps: information-analytical, creative in developing options, preliminary selection of options and selection the best option.

Schematically, the analysis process can be represented as follows:

- building a functional model of a development project;

- assessment of the significance and importance of the functions of the development project;

- cost diagnostics of functions;

- determination of the feasibility of individual functions based on their cost and relevance to goals;

- identification of possible alternatives and selection of the optimal strategy;

- the final assessment of investment attractiveness.

Since the goal of the FSA is to establish economically viable costs, attention at all stages of development focuses on the indicators of economic efficiency of the FSA. On the one hand, factors that determine the effectiveness of the use of the project are taken into account (reflected in the value of the upper limit of the development cost). On the other hand, factors that determine the value of the minimum design cost are taken into account as the basis for lowering the lower limit of cost.

Thus, the assessment of the implementation of each specific project requires a clear

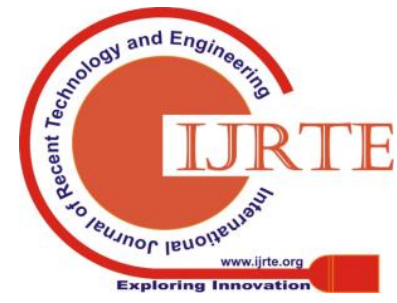


understanding of the objectives of the assessment, determination of parameters and a system of economic indicators. For AI development projects it can be implemented through the FSA, which will allow finding the optimal ratio of costs and functional content of the project.

\section{DISCUSSION}

Most risky AI development projects are expensive regardless of the uniqueness of their ideas. Therefore, before investing in a project, it is necessary to find out how relevant it is for promotion in the market.

One of the main reasons for the closure of the project at the first stages is the error in calculating the budget: innovators cannot reliably estimate the real value of their project and experience difficulties with a weak product positioning strategy for the investor. The determination of value is a key link in building mutually beneficial relations between the owner of the idea and the investor. Many aspects have to be taken into account in reaching consensus and building effective partnerships regardless of the type of financing. As a rule, it is difficult to develop a detailed plan for the implementation of the AI development project, since new factors of influence appear at each stage of implementation. Therefore, the methodology for assessing the investment attractiveness of a project should be very flexible.

If to approach the risk assessment of the AI development project systemically, given the modern development of technologies and the widespread use of Internet resources, easier ways to assess value using tools present on the modern Internet space can be found. These tools are presented in the form of sites with functions that help entrepreneurs assess the true cost and feasibility of a particular project.

Most risky AI development projects depend on personal capital, the initial savings of the founders, and venture capital. As a result, standard techniques for assessing cash flow, growth rate or discount rates do not reflect market reality. The difficulty lies in the fact that the evaluation of projects is aimed at determining their future value, while often there may not be current statistical, operational, financial, as well as retrospective data on the activities of the developer.

A key aspect of using standard methods and models for evaluating AI development projects is to evaluate the predicted effect of implementing an AI development project, the reliability, and adequacy of which are very uncertain.

Regardless of the scope for which the project is being developed, there are basic parameters for attracting investments that investors pay attention to:

- demand for the project in the market;

- risks and the possibility of minimizing them;

- the possibility of managerial control;

- the projected rate of return;

- depth of analysis of forecasts on the financial and marketing parameters of the project;

- stability of extensive and intensive project growth;

- the possibility of "exit" from the project (liquidity of a successful project, minimization of losses in case of failure, conditions for the sale of property rights to a share in the company).

\section{CONCLUSIONS}

Artificial intelligence development projects are a risky investment product, so they have to compete with other projects for limited investment resources. AI development projects have a high cost, regardless of the uniqueness of their ideas. However, most developers cannot reliably assess the real value of their project and experience difficulties with a positioning strategy.

One of the main problems of investing in the field of AI is the problem of determining the criteria for assessing the quality of investment from the position of both the investor and the recipient of the investment. The exact use of methods for assessing the effectiveness of investments in the field of AI project development is insufficient, the complex use of them without taking into account systemic aspects also does not guarantee the correctness of the calculations.

An analysis of the sources of financing risky AI development projects showed that the main resources for the development of innovative projects are venture capital companies that invest in all stages of project development. The amount of financial resources coming from business angels, crowdfunding and business accelerators is smaller and is mainly directed only to the initial stages of project implementation.

A systematic analysis of the advantages and disadvantages of various forms of investment from the point of view of the peculiarities of the development of AI projects will increase the degree of validity of decisions when choosing the investment method of both the investor and the donor company.

Evaluation of the implementation of each specific project requires a clear understanding of the methods of assessment, determination of parameters and a system of economic indicators. The idea proposed in the analysis to determine the effectiveness of the project based on the functional-cost approach allows finding the optimal ratio of costs and functional content of the project. At the same time, a condition is achieved under which the indicator of the competitiveness of future development will be higher than for analogs of the project on the market.

\section{REFERENCES}

[1] Alamsyah, A., \& Nugroho, T. B. A. (2018). Predictive modelling for startup and investor relationship based on crowdfunding platform data. Journal of Physics: Conference Series, 971, 012002.

[2] Ayukawa, M. (2012). Applying the Theory of the Firm to Examine a Technology Startup at the Investment Stage. Technology Innovation Management Review, 2(5), 23-27.

[3] Berkus, D. (2012). The Berkus Method: Valuing an Early Stage Investment. http://berkonomics .com/?p=1214

[4] Berkus, D. (2016). After 20 years: Updating the Berkus Method of valuation. https://berkonomics. com/?p=2752

[5] Cantamessa, M., Gatteschi, V., Perboli, G., \& Rosano, M. (2018) Startups' Roads to Failure. Sustainability, 10(7), 2346.

[6] Carson, S. A. (2018). Identifying Critical Risk Factors in the Decision-making Process of Angel Investors and Venture Capitalists: A Delphi Research Study. Electronic Theses and Dissertations. Paper 3360. https://dc.etsu.edu/etd/3360

[7] Damodaran, A. (2009). Valuing young, start-up and growth companies: estimation issues and valuation challenges. https://ssrn.com/abstract=1418687 http://dx.doi.org/10.2139/ssrn.1418687

[8] de Mello, F. L., \& de Souza, S. A. (2019). Psychotherapy and Artificial Intelligence: A Proposal for Alignment. Frontiers in psychology, $10,263$.

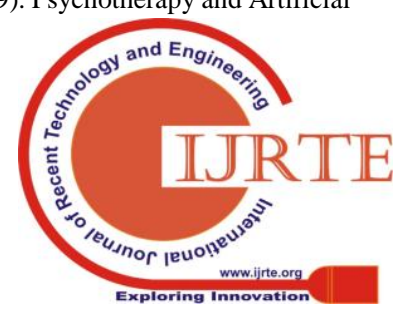


[9] Ederman, L. F., Manalova, T. S., \& Brush, C. G. (2017). Angel Investing: A Literature Review. Foundations and Trends $\mathrm{R}$ in Entrepreneurship, 13(4-5), 265-439.

[10] Kirshina, N.R., \& Lebedinsky, V.I. (2019). Features of evaluating the cost of startups. Materials for the round table "Non-standard standards: is it possible to determine the value of IP?" Library LABRATE.RU (Network resource). http://bit.ly/2XOJWnB

[11] Köhn, A. (2017). The determinants of startup valuation in the venture capital context: a systematic review and avenues for future research. Management Review Quarterly, 68.

[12] Kunitsyna, N. N., \& Khalyavskaya, T. V. (2016). Methods for assessing the pre-investment value of startups that have not reached the level of profitability. Scientific and Technical Journal of St. Petersburg State Polytechnical University, 4 (246), 292-301

[13] Loktionova, Yu.N. (2017) Financial analysis of investment projects: basic directions and methods of carrying out. Social policy and sociology, 16(2(121), 47-55.

[14] Loktionova, Yu.N., \& Yanina, O.N. (2019) Approaches to measuring innovation in the economy. Social policy and sociology, 18(1 (130), $32-41$

[15] Mannar, K. (2019). The ROI of AI https://www.accenture.com/us-en/insights/artificial-intelligence/roi-ar tificial-intelligence

[16] Mezentsev, Yu.A., \& Preobrazhenskaya, T.V. (2003). Functional cost analysis. Tools and models: textbook. Allowance. Novosibirsk: NSTU, 122.

[17] OECD (2018). Private Equity Investment in Artificial Intelligence. OECD Going Digital Policy Note, OECD, Paris, www.oecd.org/going-digital/ai/private-equity-investment-in-artificialintelligence.pdf

[18] Payne, B. (2011). Valuations 101: The Dave Berkus Method. http://blog.gust.com/248/

[19] Payne, B. (2011b). Valuations 101: The Venture Capital Method. http://blog.gust.com/startup-valuations-101-the-venture-capital-meth od/

[20] Payne, B. (2011c). Valuations 101: The Risk Factor Summation Method

http://blog.gust.com/valuations-101-the-risk-factor-summation-metho d/

[21] Payne, B. (2017). Scorecard Valuation Methodology: Establishing the Valuation of Pre-revenue, Start-up Companies. http://etd.lib.metu.edu.tr/upload/12621330/index.pdf

[22] Saint-Pierre, J. (2017). A Simple Test of the Value of Artificial Intelligence (AI) for Investments. https://ssrn.com/abstract $=3071052$ or http://dx.doi.org/10.2139/ssrn.3071052

[23] Zhong, H., Liu, C., Zhong, J., \& Xiong, H. (2018). Which startup to invest in: a personalized portfolio strategy. Annals of operations research, 263(1-2), 339-360. 